\title{
The Effects of Nutrition Education on Food Habits of the Elderly
}

\author{
Yanfeng Liü, a \\ ${ }^{1}$ Medical School of Yan'an University, Yan'an, Shaanxi, 716000 \\ ${ }^{a}$ email
}

Keywords: Health education, Residents, Food habits, Stages of change

\begin{abstract}
Object: To explore the effects of health education based on the Trans-theoretical Model and Stages of Change for Residents of elderly on food habits. Method: Eighty urban residents with age $\geqslant 60$ years were recruited as participants. After baseline survey was carried out with self-edited questionnaires, health education on food habits was conducted to the participants. Three months later the second survey was carried out with the same questionnaire. Results: The nutritional knowledge of objects had increased significantly after health education $(\mathrm{P}<0.01)$. The distributions of stages of change in objects of high fat intake, pickles intake and salt intake and low milk intake were significantly different with that of them before health education $(\mathrm{P}<0.05)$, while that of objects of low fruits and vegetables intake was not significantly different $(\mathrm{P}>0.05)$. Conclusions: Health education carried out according to the different stages of change of objects can effectively alter the food habits.
\end{abstract}

\section{Introduction}

The 2004 survey on the nutritional and health status of Chinese residents showed that the nutritional status of Chinese residents had been greatly improved. However, there were still serious malnutrition problems: First, the inadequate intake of certain dietary nutrients prevented the improvement of population quality . Iron, vitamin A and other micronutrient deficiencies, milk, soy products intake is still low urban and rural residents in China is still widespread problems. Second, over-nutrition causes a sharp increase in chronic diseases in modern society. At present, the prevalence rate of adult hypertension in China is $18.8 \%$, moderate and mild hypertension is $85 \%$; the prevalence of diabetes in adults is $2.6 \%$; the prevalence of diabetes in metropolises above 20 years old rises to 6.4\%; adult blood lipids The prevalence rate of overweight and obesity was $22.8 \%$, the obesity rate was $7.1 \%$, the prevalence rate of overweight and obesity was $7.1 \%$, and the prevalence rate of overweight and obesity was $22.8 \%$. The prevalence rate of overweight and obesity was $18.6 \%$ Adult overweight and obesity rates were as high as $30.0 \%$ and $12.3 \%$, respectively, compared with 1992 National Nutrition Survey data, adult overweight rate increased by $39 \%$, obesity rate increased by $97 \%$, due to overweight base, the future prevalence of obesity will There is a substantial increase [1].

China's residents in the new era of these health problems and their eating habits are very closely linked. Dietary high-energy, high-fat and less physical activity and overweight, obesity, diabetes and dyslipidemia are closely related to the development of high-salt diet and high blood pressure is closely related to the risk of drinking [4]; drinking and hypertension And dyslipidemia is closely related to the risk of illness. In particular, should be noted that the most fat intake, the least physical activity of people suffering from the above chances of the most chronic diseases. Fresh vegetables, fruit intake or lack of regular intake of pickled food or spicy food will increase the risk of esophageal cancer [5], smoking, fatigue, frequent consumption of sweets and barbecue can increase the risk of Hp infection, lifestyle and regular consumption Acidic foods, green vegetables and green tea has anti-Hp infection [6]; increase milk and soy products intake can also reduce women's symptoms of menopausal syndrome [7].

China has entered the elderly society, so gradually improve health education through eating habits of older residents, has become one of the important tasks of preventive medicine research. 
However, the majority of domestic studies are investigative studies, intervention studies rarely. Investigative research is mainly on the general population of eating habits [8] or a variety of diseases (such as high blood pressure, diabetes, etc.) and the relationship between eating habits survey to determine the current poor eating habits and its relationship with the The relationship between various diseases. The main adverse eating habits of urban residents are mainly urban residents' dietary structure is not reasonable, livestock and oil consumption is too much, cereal consumption is low; pickled foods and excessive salt intake; milk and soy products , Fresh fruit, vegetable intake is insufficient. But there is little research on the eating habits of urban elderly residents. Intervention research mostly intervened on nutrition knowledge and attitudes. Health education was mainly carried out on knowledge propaganda. Behavior interventions were mainly on children, primary and middle school students and college students. The main means of intervention is the use of words, pictures, videos, lectures and other means of publicity and education and telephone counseling.

There are many theories about health-related behavioral change, such as KABP, HBM, HPM, TTM, and so on. TTM (The Transtheoretical model and stages of change) was proposed by Prochaska [9] in the early 1980s. Intervention studies, initially on tobacco use, later covered a wide range of areas including alcohol and substance abuse, dietary behavior, Life style, AIDS prevention, compliance behavior, unplanned pregnancy intervention and so on. It is one of the most widely used behavioral change theories in the world. Prochaska argues that human behavior change is not a one-time event, but rather a gradual and continuous process. Effective behavioral change should be a gradual process that spans five stages: the preconception phase (without considering changing dietary habits) (In the next 6 months, people are planning to change the bad eating habits stage), the preparation phase (in the next 30 days, people will change the bad eating habits, and has done some preparatory work stage), the stage of the intention, The stage of action (which has begun to change bad eating habits in the last six months), the maintenance phase (people have changed bad eating habits). TTM is a dynamic model of comprehensive behavioral change. The traditional one-time behavioral intervention model is a phased intervention model, which provides targeted behavioral support techniques according to the needs of behavior changeers. It is widely used in clinical and community behavior interventions Effective strategies and methods [10]. Therefore, this study uses the existing health education methods, the behavior of the phased transformation theory as a guide, in the community for the elderly urban residents in various forms of health education to change its existing bad eating habits, while for large-scale The intervention study provides a reference.

\section{Objects and Methods}

According to the building number in the residential area of Bao'an District, Yan'an City, 80 subjects aged 60 or older were selected as the study subjects by systematic sampling method.

According to the different stages of the behavior change of the study subjects, a variety of health education methods were adopted.

Interactive expert lectures: once every weekend, every 60 minutes, a total of 6 times. It includes a balanced diet, the importance of good eating habits, the relationship between eating habits and disease, how to assess current eating habits, how to change existing bad eating habits, and how to maintain a healthy diet.

Distribution of publicity materials: In the expert lectures and food consultation issued "Chinese residents dietary guidelines", "Chinese residents balanced diet pagoda" and other promotional materials.

Questionnaire survey. Before the intervention, questionnaires were retrieved by self-administered questionnaires by investigators trained by the system. Three months after the intervention, the same investigator used the same scale to conduct a second survey of the subjects.

Self-made questionnaire, including: (1) general situation: gender, age, occupation, hobbies and so on. (2) diet, including: animal fat, pickled foods, salt, milk, fruit and vegetable intake. Animal fats are lard, tallow, sheep oil, chicken fat and all kinds of animal fat, the survey is divided into an average weekly intake of 0 meals, 1 to 3 meals, 4 to 6 meals, 7 to 9 meals And more than 10 meals 
in several cases, the average weekly intake of animal fat 4 to 6 meals and more than 4 to 6 meals are regular intake; pickled foods, including preserved meat, fish, vegetables, Average daily intake of 4 to 6 meals and more than 4 to 6 meals are regular intake; salt daily intake of more than $7 \mathrm{~g}$ were high-salt diet; fruit and vegetables intake of more than $500 \mathrm{~g}$ per day for intake ; Milk intake of an average of more than $200 \mathrm{ml}$ per day for the intake. (3) The stages of the process of behavior change: residents in the former intent stage, intention stage, preparation stage, action stage, maintenance stage of the status quo. (4) knowledge of the extent of knowledge of a reasonable diet.

After all the information is correct, enter the computer, using SPSS16.0 software package for statistical operations. Measurement data using mean and standard deviation described by t test for comparison; count data to describe the percentage of before and after the intervention with $\chi^{2}$ test.

\section{Results}

80 questionnaires were distributed before the intervention, 76 questionnaires were returned, and 74 were valid responses. The effective response rate was $92.5 \%$. After the intervention, the second questionnaires were conducted in March, 74 questionnaires were sent out, 74 questionnaires were returned, and the effective responses were 74 . The effective response rate was $100 \%$.

Among the 74 subjects, $45.9 \%$ were males and 54.1\% were females; the oldest were 70 years old and the youngest 60 years old, the average age was $66.4 \pm 1.36$ years; the distribution of the former occupation was $32.4 \%$, the teacher was $10.8 \%$, $16.2 \%$ for civil servants, $8.1 \%$ for medical personnel, $12.2 \%$ for unemployed persons, $12.2 \%$ for tradesmen and $8.1 \%$ for staff. Distribution of education: $10.9 \%$ of primary and illiteracy, $21.6 \%$ of junior high school, $37.8 \%$ of high school and secondary school, college and above is $29.7 \%$.

$36.5 \%$ of the residents often intake of animal fats, of which $12.2 \%$ of the residents of the average daily intake of animal fat a meal or more; $79.7 \%$ of the residents of the intake of preserved foods, 9.5\% of the residents of the average daily intake of pickled food Of the population; $55.4 \%$ of the residents daily salt intake is greater than $7 \mathrm{~g}, 37.8 \%$ of the residents drink about $200 \mathrm{ml}$ of milk a day; $54.1 \%$ of the residents eat about $500 \mathrm{~g}$ of fruit and vegetables per day (see Table 1 ). Among all kinds of food, the percentage of males inhaling animal fat was higher than that of female $\left(\chi^{2}=4.957, \mathrm{P}=\right.$ $0.026)$. $(\mathrm{X} 2=1.808, \mathrm{P}>0.05)$; the percentage of intake of milk did not increase significantly $(\chi 2=$ $7.301, \mathrm{P}=0.063)$, and the percentage of intake of animal fat was not significantly decreased $(\chi 2=$.

Table 1 Households' Food Intake (\%)

\begin{tabular}{|c|c|c|c|c|c|c|c|}
\hline \multirow[b]{2}{*}{ Types of Diet } & \multicolumn{2}{|c|}{ Gender } & \multicolumn{4}{|c|}{ Educational level } & \multirow[b]{2}{*}{ total } \\
\hline & $\begin{array}{l}\text { Men } \\
\text { wome }\end{array}$ & & $\begin{array}{l}\text { Illiteracy } \\
\text { and } \\
\text { primary } \\
\text { school }\end{array}$ & $\begin{array}{l}\text { junior } \\
\text { high } \\
\text { school }\end{array}$ & $\begin{array}{c}\text { High } \\
\text { school and } \\
\text { secondary } \\
\text { school }\end{array}$ & $\begin{array}{c}\text { College } \\
\text { and above }\end{array}$ & \\
\hline Animal fat & 50.0 & 25.0 & 50.0 & 43.75 & 35.71 & 27.27 & 36.5 \\
\hline Pickled foods & 82.4 & 79.4 & 75.5 & 81.25 & 78.57 & 81.81 & 79.7 \\
\hline High-salt food & 77.5 & 80.0 & 62.5 & 56.25 & 53.57 & 54.5 & 55.4 \\
\hline milk & 35.0 & 50.0 & 16.7 & 18.8 & 50.0 & 54.5 & 41.7 \\
\hline $\begin{array}{l}\text { fruit and } \\
\text { vegetable }\end{array}$ & 47.1 & 60.0 & 50.0 & 56.25 & 53.6 & 54.5 & 54.1 \\
\hline
\end{tabular}

The score of reasonable dietary knowledge was $18.85 \pm 4.50$ before intervention and $25.68 \pm$ 0.73 after intervention. Compared with before intervention, the score of reasonable dietary knowledge was significantly increased after intervention $(\mathrm{t}=12.188, \mathrm{P}<0.001)$.

Pre-intervention residents in the distribution of behavior at various stages of the transition

$27.0 \%$ of the inhabitants of the intake of animal fats were in the preconscious stage, and no consideration was given to the time when the intake of animal fats was no longer regular. 2.7 The dietary habits of the residents were distributed in different stages of transformation. \% Of the population in the intent stage, considered within the past 6 months to start no longer frequent intake of animal fat; $1.4 \%$ of residents in the preparatory stage, ready to start in the past $30 \mathrm{~d}$ infrequent animal fat; Residents in the action stage, nearly 6 months is no longer frequent intake of animal fats; 
$1.4 \%$ of the residents are in the maintenance phase, they have changed the regular intake of animal fat in poor eating habits. Residents of other eating habits in the distribution of the various stages, see Table 2.

The distribution of dietary habits of residents after the intervention in the various stages of behavior change

After the intervention, the dietary habits of all kinds of residents in different stages of transformation are distributed in the intake of animal fat in the residents, $6.8 \%$ of the residents in the pre-intended stage, has not yet considered when to start no longer frequent intake of animal fat ; $8.1 \%$ of the residents in the intent stage, considering the past 6 months to start no longer frequent intake of animal fat; $9.5 \%$ of the residents in the preparatory stage, ready to start in the past 30d infrequent animal fat; \% Of the population are in the action phase and no more frequent intake of animal fats in the last 6 months; $2.7 \%$ of the population is in the maintenance phase, and they have changed the bad dietary habits of regular intake of animal fats. The distribution of other dietary habits of residents in various stages, see Table 3.

Table 2 the distribution of dietary habits of residents before the intervention in each stage of behavior change

\begin{tabular}{|c|c|c|c|c|c|c|c|c|c|c|}
\hline \multirow[t]{2}{*}{ Behavioral stage } & \multicolumn{10}{|c|}{$\begin{array}{l}\text { Pre - Intention Phase Intention Phase Preparation Phase Action Phase } \\
\text { Maintenance Phase }\end{array}$} \\
\hline & \multicolumn{10}{|c|}{ Example Number Percentage (\%) } \\
\hline Animal fat often & 20 & 27.0 & 2 & 2.7 & 1 & 1.4 & 3 & 4.1 & 1 & 1.4 \\
\hline intake & 42 & 56.8 & 3 & 4.1 & 7 & 9.5 & 3 & 4.1 & 2 & 2.7 \\
\hline Pickled food intake & 17 & 23.0 & 5 & 6.8 & 7 & 9.5 & 6 & 8.1 & 6 & 8.1 \\
\hline High-salt diet & 15 & 20.3 & 4 & 5.4 & 4 & 5.4 & 3 & 4.1 & 2 & 2.7 \\
\hline Milk intake less & 12 & 16.2 & 6 & 8.1 & 4 & 5.4 & 14 & 18.9 & 4 & 5.4 \\
\hline $\begin{array}{l}\text { Fruits, vegetables, } \\
\text { less intake }\end{array}$ & & & & & & & & & & \\
\hline
\end{tabular}

Table 3 the distribution of dietary habits of residents after the intervention in various stages of behavior change

\begin{tabular}{|c|c|c|c|c|c|c|c|c|c|c|}
\hline \multirow[t]{2}{*}{ Behavioral stage } & \multicolumn{10}{|c|}{$\begin{array}{l}\text { Pre - Intention Phase Intention Phase Preparation Phase Action Phase } \\
\text { Maintenance Phase }\end{array}$} \\
\hline & \multicolumn{10}{|c|}{ Example Number Percentage (\%) } \\
\hline Animal fat often & 5 & 6.8 & 6 & 8.1 & 7 & 9.5 & 6 & 8.1 & 2 & 2.7 \\
\hline intake & 9 & 12.2 & 12 & 13.5 & 14 & 18.9 & 8 & 13.5 & 5 & 6.8 \\
\hline Pickled food intake & 4 & 5.4 & 12 & 16.2 & 10 & 13.5 & 10 & 13.5 & 8 & 10.8 \\
\hline High-salt diet & 4 & 6.8 & 5 & 5.4 & 6 & 8.1 & 8 & 10.8 & 5 & 6.8 \\
\hline Milk intake less & 3 & 4.1 & 8 & 10.8 & 7 & 9.5 & 16 & 21.6 & 6 & 8.1 \\
\hline $\begin{array}{l}\text { Fruits, vegetables, } \\
\text { less intake }\end{array}$ & & & & & & & & & & \\
\hline
\end{tabular}

Compared with the pre-intervention, the distribution of lipid intake in the animal behavior changed significantly $(\chi 2=16.820, \mathrm{P}=0.002)$ after the intervention; the distribution of the pickled food intake changed significantly in each stage of behavior change $(\mathrm{X} 2=16.820, \mathrm{P}<0.001)$. The distribution of behavior changes in high-salt diets changed significantly $(\chi 2=12.665, \mathrm{P}=0.013)$; the changes in the behavioral changes were significant $(\mathrm{X} 2=10.438, \mathrm{P}=0.034)$. However, there was no significant change in the distribution of behavioral changes in the two groups $(\chi 2=7.037, \mathrm{P}$ $=0.314)$.

\section{Discussion}

In the elderly urban residents, there are the following is not conducive to healthy eating habits: dietary structure is irrational, livestock and oil consumption too much; pickled foods and high salt intake of excessive food; milk and other quality protein and fresh fruit Vegetable intake is too small. This is the 2004 survey of Chinese residents nutrition and health status is basically the same [1]. Male eating behavior worse than women, male residents may be less concerned about the details of life, more likely to ignore their own bad eating behavior. In addition, the results showed that with 
the increase of education level, the increase of milk intake was not statistically significant $(\mathrm{P}=$ 0.063), and Jiang Xuefang [7], Xu Liangwen [8] found that the higher the level of milk intake The higher the amount of inconsistent, which may be due to the small sample size of the study. Low level of education for residents of the cultural level of restrictions on the outside world to accept education is slow, the lack of a certain nutritional knowledge.

Health education can significantly improve the knowledge of urban residents about a reasonable diet, which is consistent with many studies [11]. Some residents have knowledge of a reasonable diet, but there are still some residents insist on changing their eating habits, which shows that the growth of health knowledge is not always accompanied by changes in bad behavior. This is the result of $\mathrm{Xu}$ Wenjuan's research on the anemia of middle school students. Indicating that the increase in knowledge is to produce a necessary condition for behavior change, rather than sufficient conditions, can not lead to inevitable behavior changes.

There are five different stages in the change of eating habits of elderly urban residents, and the ladder-like shows that people's bad eating habits change, not simply from the left to the right, from one side to the other, but need to cross A series of stages. After health education, the distribution of animal fat intake, salted food intake, high - salt diet and milk intake were significantly changed ( $\mathrm{P}$ $<0.05$ ), and the change of the change More beneficial to the occurrence of effective behavioral changes, indicating that the stage of the study carried out in a variety of ways of health education can effectively promote behavioral change; and fruit and vegetable intake of those less in health education compared to before There is no significant difference, probably because of eating habits affected by social, economic, cultural and other factors, and interventions failed to change the impact of these factors.

Residents who are in different stages of behavioral change can take the following measures: (1) For the residents who are in the pre-intention stage and the intention stage, they can strengthen the education on changing the results of bad eating habits, promote a balanced diet, Diet habits, in order to enhance their awareness, and promote their behavior attitudes change. (2) For residents in the preparatory stage, they can provide a balanced diet, a good diet of the feasibility analysis, to provide a gradual change in dietary behavior of the program to increase their behavior change beliefs. (3) For the residents in the stage of action and maintenance, they should strengthen their confidence in balanced diet, good eating habits, increase their perseverance, support social forces and consolidate the results of behavior transformation.

\section{Conclusions}

Bad eating habits in China caused by a sharp increase in chronic diseases in modern society, in which the elderly urban residents in the incidence of these diseases is increasing, and gradually improve health education through the elderly urban residents eating habits, become an important task of preventive medicine research one.

According to the different stages of the study subjects to take a variety of health education methods and measures can effectively increase the elderly urban residents a reasonable diet instruction to change their poor eating habits, improve health education effect.

In this study, because the sample size is too small, the study needs to be more people to further verify the effectiveness of health education; in the process of health education, the subjects may also accept the impact of other than interventions. Therefore, in future studies, the need to set up a control group to control a variety of confounding factors. Due to the limitations of the study, the second survey was conducted three months after health education. Because of the time required for human behavior change, the effect of health education can not be revealed and measured in a short time. Therefore, For a long time to conduct further investigation. In addition, the intervention study to increase the intake of fresh fruits and vegetables in elderly urban residents is also a future research issue. 


\section{Acknowledgements}

2016 Scientific Research Project of Shaanxi Department of Education (16JK1867)

Yan'an University 2015 research projects (YDK2015-70)

Yan'an University Research Project for Continuing Education in 2015 (YDJQ2015-08)

\section{References}

[1] Cheng Yiyong. Chinese residents nutritional status and related health problems. Chinese food and nutrition .2005,1: 6-8

[2] Huang Li, Wang Wenju, Wu Sufen. Dietary habits and lifestyles and the incidence of impaired glucose tolerance in the elderly study. Journal of Nursing .2004,21 (4): 4-6

[3] Zhong Li, Zhang Qin, He Dajun. Dietary behavioral analysis in obese and diabetic patients. Chinese Journal of Physical Medicine and Rehabilitation. 2000, 22 (6): 343-345

[4] Chen Lin, Pan Feng, Qian Yaqiong .Hypertension and eating habits of Jiangxi Medical, 2005,40 (5): 278-279

[5] Chen Kailin, Yin Houyuan, Yi Datong. Tobacco, alcohol and eating habits in the incidence of esophageal cancer in low-incidence relationship. Chinese tumor, 1995,4 (7): 7-9.

[6] Zhang Linhan, Yu Gang, Dai Xiang. Health habits and Helicobacter pylori infection correlation study, 2005,14 (8): 699-700

[7] Jiang Xuefang, Tian Fenglian, Zhao Qingguo. Perimenopausal women's eating habits and perimenopausal syndrome in the relationship between China's Maternal and Child Health Care .2005, 20 (6): 667-669.

[8] Xu Liangwen, Ma Haiyan, YANG Ting-zhong.Research on the status quo of the dietary behavior change of the residents.Preliminary Journal of Preventive Medicine 2004, 38 (3): 179-181

[9] Prochaska JO, Redding CA, Evere KE. The transtheoretical model and stages if change. In: Health behavior and health education, San Francisco: Jossey Bass Publishers, 1997 61-83

[10] Yang Ting-Zhong, Yu Wenping, Huang Li. A Method And Method of Behavioral Change: An Introduction Of Theoretical Model of Performing Stage Transition. Chinese Journal of Behavioral Science, 2002, 11 (3)

[11] Yu Jie, Yu Youjuan, Gu Qun. Intervention measures to improve the community middle-aged crowd reasonable nutrition cognition rate effect. Shanghai Journal of Preventive Medicine 2005, 17 (1): 41-42 